\section{ORIGINAL RESEARCH}

\author{
L.G. Merckel \\ J. Van der Heijden \\ L.M. Jongen \\ H.W. van Es \\ M. Prokop
}

A. Waaijer

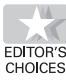

\title{
Effect of Stenting on Cerebral CT Perfusion in Symptomatic and Asymptomatic Patients with Carotid Artery Stenosis
}

BACKGROUND AND PURPOSE: The introduction of CAS has led to increased treatment of both symptomatic and asymptomatic patients with internal carotid stenosis. This study was performed to compare the effect of stent placement on cerebral perfusion in symptomatic and asymptomatic patients using CT perfusion.

\begin{abstract}
MATERIALS AND METHODS: We included 45 patients with carotid artery stenosis of $\geq 70 \%$ who underwent arterial stent placement. Thirty-one patients were treated because of symptoms; 14 asymptomatic patients were treated before coronary artery bypass grafting. Patients underwent CTP before and after stent placement. We calculated MTT, CBV, and CBF, and derived relative numbers that compared treated with untreated hemispheres: ratios of CBV and CBF and difference in MTT. We compared the effect of carotid stent placement on cerebral perfusion in symptomatic and asymptomatic patients.
\end{abstract}

RESULTS: All perfusion parameters changed significantly after treatment in symptomatic patients: rCBF increased from 0.81 to $0.93(P<.001)$, rCBV decreased from 1.02 to $0.95(P<.05)$, and dMTT decreased from 1.29 to $0.14(P<.001)$. In asymptomatic patients only, rCBF changed significantly with an increase from 0.92 to $1.03(P<.05)$. When we compared symptomatic and asymptomatic patients before treatment, rCBF in symptomatic patients was significantly lower. The decrease of rCBV after treatment in symptomatic patients resulted in a significantly lower value than in asymptomatic patients.

CONCLUSIONS: Carotid artery stent placement improves blood flow in the affected hemisphere in symptomatic and asymptomatic patients. CBF before treatment is more strongly impaired in patients with symptomatic carotid stenosis. Compensatory hyperemia on the symptomatic side before treatment $(\mathrm{rCBV}>1)$ turns into hypoxemia after treatment, suggesting impaired autoregulation in these patients.

ABBREVIATIONS: CABG = coronary artery bypass grafting; CAS = carotid artery stenting; $\mathrm{CEA}=$ carotid endarterectomy; $\mathrm{dMTT}=$ difference in mean transit time; $\mathrm{rCBF}=$ ratio of cerebral blood flow; rCBV = ratio of cerebral blood volume

$\mathbf{R}$ isk of ischemic stroke increases with the severity of stenosis in both symptomatic and asymptomatic patients with internal carotid artery stenosis. ${ }^{1}$ The introduction of CAS as an alternative to surgery has led to a renewed search for risk factors of stroke to improve the selection of patients who would profit most from such an intervention. Several studies compared carotid plaque composition from symptomatic and asymptomatic patients to understand the underlying mechanisms. ${ }^{2}$

A severe carotid artery stenosis may cause reduced perfusion pressure and will, therefore, influence cerebral hemodynamics. Collateral circulation, on the other hand, can maintain normal cerebral perfusion pressure and normal flow in many patients with carotid artery stenosis. ${ }^{3}$ In symptomatic patients with severe carotid stenosis and occlusion, ${ }^{4-6}$ the association of cerebral hemodynamics with the risk of stroke has

Received March 11, 2011; accepted after revision May 26.

From the University Medical Center Department of Radiology (L.G.M., L.M.J., M.P.), Department of Cardiology (J.V.H., H.W.E.) Utrecht, the Netherlands; and St. Antonius Hospital (J.v.d.H., H.W.v.E.), Nieuwegein, the Netherlands.

Paper previously presented at: Annual Meeting of the European Congress of Radiology, March 4-8, 2010; Vienna, Austria.

Please address correspondence to L.G. Merckel, MD, Department of Radiology, University Medical Center Utrecht, Heidelberglaan 100, E01.132, 3584 CX Utrecht, the Netherlands; e-mail: L.G.Merckel-2@umcutrecht.nl

http://dx.doi.org/10.3174/ajnr.A2757 been described. In asymptomatic patients with severe carotid stenosis, less proof of this association is available. However, Silvestrini et $\mathrm{al}^{7}$ showed that patients with severe asymptomatic carotid artery stenosis might be hemodynamically compromised. Furthermore, Soinne et $\mathrm{al}^{8}$ showed that early and late $\mathrm{CBF}$ postoperatively are slightly higher than preoperative values in the ipsilateral and contralateral arteries in asymptomatic patients, though the differences before and after treatment were minor.

Treatment of internal carotid artery stenosis of $\geq 70 \%$ in symptomatic patients is generally accepted, but on average, 6 patients have to undergo CEA to prevent 1 stroke. ${ }^{9}$ The benefit of treatment in asymptomatic patients is still controversial because substantially more patients need to be treated to prevent 1 stroke. The annual risk of stroke in asymptomatic patients with a stenosis of $60 \%-99 \%$ is estimated to be $3.2 \% .{ }^{10}$ Despite this low annual risk, several trials indicate the benefit of treatment in asymptomatic patients. ${ }^{11-13}$

The introduction of CAS has led to increased treatment of both symptomatic and asymptomatic patients, though the benefit of stent placement has not been proved for all indications. CTP has been used to evaluate the effect of stent placement in symptomatic patients with carotid artery stenosis of $\geq 50 \%$, showing significant improvement of CTP parame- 
ters. ${ }^{14,15}$ Currently, however, it is not completely clear whether perfusion will also improve in asymptomatic patients after CAS.

Therefore, in this study, we compare the effect of stent placement on cerebral perfusion in symptomatic and asymptomatic patients with internal carotid artery stenosis by using CTP analysis.

\section{Materials and Methods}

\section{Patients}

All symptomatic patients were participants in the International Carotid Stenting Study, a randomized controlled trial in which CEA and stent placement are compared in patients with a symptomatic stenosis (www.cavatas.com, ISRCTN 25337470). Patients with an asymptomatic stenosis were diagnosed and treated during the work-up for CABG. Inclusion of all patients was based on the presence of carotid artery stenosis of $\geq 65 \%$ measured on duplex or CTA according to the North American Symptomatic Carotid Endarterectomy Trial criteria, and accessibility for carotid stent placement (ie, no tortuous anatomy proximal or distal to stenosis). Subjects had to be independent in daily life (modified Rankin Scale score of $\leq 3$ ), with no history of previous ipsilateral CEA or radiation therapy.

Both symptomatic and asymptomatic patients were clinically evaluated by an independent neurologist before the procedure, during the procedure, and immediately afterward. The mean time from symptom onset to treatment was 44.3 days in symptomatic patients. All included patients underwent CAS. Exclusion criteria were a contralateral stenosis of $>50 \%$ and the presence of contraindications for CTA, such as renal failure or contrast allergy. The degree of stenosis was measured with duplex sonography in combination with CTA for symptomatic patients. In asymptomatic patients, it was assessed by using duplex sonography. None of the patients had significant stenoses in the intracranial vasculature on CTA.

From October 2003 until April 2006, a CTP study was performed in 59 symptomatic patients who underwent CAS. Twenty-eight patients were excluded from analysis: Nineteen patients had a contralateral stenosis of $>50 \%$. Another 9 patients were excluded due to a missing pre- or posttreatment scan or technical problems with contrast administration, motion artifacts, or problems during postprocessing. Consequently, our selection process yielded 31 symptomatic patients for further analysis.

From October 2006 till January 2009, a CTP study was performed in 27 asymptomatic patients. Thirteen patients were excluded from the analysis: Six patients had a contralateral stenosis of $>50 \%$ and 7 patients were excluded due to a missing pre- or posttreatment scan or technical problems with contrast administration, motion artifacts, or problems during postprocessing. Consequently, this process yielded 14 asymptomatic patients for further analysis.

In both groups, we strived for a scan within 1 week before treatment and 1 month after CAS. The medical ethics committees of the 2 hospitals participating in this study had given approval for this study. Written informed consent was obtained from all patients.

\section{CAS Procedure}

Stents and other devices used for CAS were chosen at the discretion of the interventionist but had to have a CE mark. The protocol recommended that a cerebral protection device be used, but this was not mandatory. On the basis of the degree and shape of the stenosis, it was decided whether pre- and postdilation was required. A combination of aspirin and clopidogrel to cover stent-placement procedures was recommended. ${ }^{16}$

\section{CTP}

Imaging Protocol. In both institutions, a Philips Healthcare (Best, the Netherlands) multisection CT scanner was used. CTP data were acquired at the level of the basal ganglia: using a 16-detector row (Mx8000 IDT), 40-detector row (Brilliance-40), or 64-detector row (Brilliance-64) scanner; all from Philips Medical Systems, Cleveland, Ohio. The cycle time was 2 seconds, resulting in 30 images acquired during 60 seconds, which has shown to result in accurate perfusion data. ${ }^{17}$ For an optimum signal intensity-to-noise ratio, we used a low-kilovolt(peak) technique in combination with $150 \mathrm{mAs} .{ }^{18}$ For the 16-detector row scanner, we had 2.4-cm coverage (collimation $8 \times 3 \mathrm{~mm}$, reconstructed in two $12-\mathrm{mm}$ slabs). With the introduction of 40-detector row and 64-detector row, this improved to 4 -cm coverage (collimation of $32 \times 1.25 \mathrm{~mm}$, images were reconstructed in 4 adjacent slabs of $10 \mathrm{~mm}$ or $64 \times 0.625 \mathrm{~mm}$ collimation reconstructed in 8 slabs of $5 \mathrm{~mm}$ ). For all perfusion scans, a bolus injection of $40 \mathrm{~mL}$ of contrast with an iodine concentration of $300 \mathrm{mg} \mathrm{I} / \mathrm{mL}$ iopromide (Ultravist 300; Schering, Berlin, Germany) was administered at 5 $\mathrm{mL} / \mathrm{s}$, followed by a $40-\mathrm{mL}$ saline chaser bolus at $5 \mathrm{~mL} / \mathrm{s}$ by using a power injector with a dual-head system. The scans were obtained at the level of the basal ganglia, $3 \mathrm{~cm}$ above the dorsum sellae, with the scan angle set parallel to the orbitomeatal line to avoid direct radiation exposure to the eye lens. Although symptomatic and asymptomatic patients were scanned in different hospitals, imaging protocols of all scans were equal.

Data Analysis. CTP data were transferred to a postprocessing workstation (Extended Brilliance Workspace, Philips Healthcare), on which CBV, MTT, and CBF were calculated by using a deconvolution technique. ${ }^{19}$ Postprocessing of CTP data was performed in a standardized way. The anterior cerebral artery was used as the arterial input function; the superior saggital sinus was used as the venous output function. Regions of interest were drawn according to a standardized method and guided by an expert opinion for each patient individually. A vascular pixel elimination method was used to exclude vascular pixels and, therefore, avoid over- or underestimation of perfusion data. We excluded regions of prior infarction in regions of interest. To quantify changes in perfusion parameters before and after stent placement, we matched 2 slabs close to the level of the basal ganglia of the pretreatment CTP examination to 2 corresponding slabs at the same level on the posttreatment CTP examination. A region of interest corresponding to the cortical flow territory of the middle cerebral artery of both hemispheres according to the maps of Damasio $^{20}$ was manually outlined on each slab (Fig 1).

The deconvolution technique used provides estimates of absolute perfusion data for each pixel in treated and untreated hemispheres; CBV expressed as milliliter/100 g tissue, MTT expressed in seconds, and CBF expressed in milliliters/100 g tissue/min. Because absolute cerebral perfusion is known to depend also on extracerebral factors as well, ${ }^{21}$ we chose to normalize measured values in the treated hemisphere to those in the untreated hemisphere. As a relative measure for MTT, we chose the absolute difference in MTT values between the treated and untreated hemispheres. For relative $\mathrm{CBV}$ and relative $\mathrm{CBF}$, the ratios of the treated to the untreated hemispheres were calculated. For each CTP examination, the relative CTP data were averaged over the 2 adjacent slabs included in the evaluation.

First, we analyzed symptomatic and asymptomatic patients separately, comparing relative pre- and posttreatment values by using a 


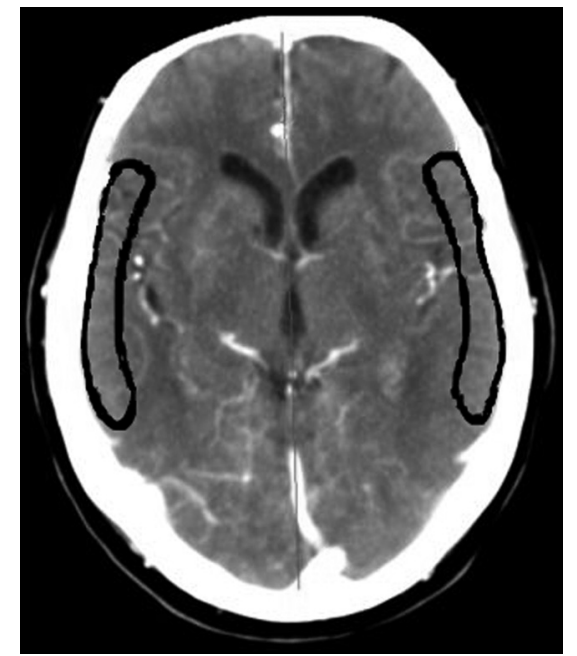

Fig 1. Manual outlining of middle cerebral artery territory on transverse CT sections, according to the maps of Damasio. ${ }^{20}$

paired $t$ test and the Wilcoxon signed rank test for 2 related samples. Subsequently, we analyzed differences between symptomatic and asymptomatic patients for both pre- and posttreatment values by using the Mann-Whitney $U$ test for 2 independent samples. Statistical analysis was performed by using the Statistical Package for the Social Sciences, Version 15.0 (SPSS, Chicago, Illinois). A $P$ value $<.05$ was considered statistically significant.

\section{Results}

We analyzed 31 symptomatic and 14 asymptomatic patients with both pre- and posttreatment CTP scans. Patient characteristics are shown in Table 1.

\section{Change of CTP after Treatment}

In symptomatic patients, $\mathrm{rCBF}$ increased from 0.81 to 0.93 $(P<.001)$, rCBV decreased from 1.02 to $0.95(P<.05)$, and dMTT decreased from 1.29 to $0.14(P<.001)$ after treatment.

In asymptomatic patients, only rCBF changed significantly after treatment: It increased from 0.92 to $1.03(P<.05)$. The decrease in dMTT from 0.56 to 0.06 did not reach significance $(P=.081)$. There was no significant change in rCBV (Table 2 and Fig 2).

\section{Comparison of Symptomatic and Asymptomatic Patients}

Before treatment, we found a significantly lower rCBF $(0.81$ versus $0.92, P<.005)$ in symptomatic patients. dMTT was almost significantly higher in symptomatic patients ( 1.29 versus $0.56, P=.061$ ), and $\mathrm{rCBV}$ showed no significant difference before treatment.

After treatment, only rCBV showed a significant difference: rCBV was significantly lower in symptomatic patients $(0.95$ versus $1.05, P<.005)$. The difference in dMTT was not significant $(0.14$ versus $0.06, P=.57)$, while $\mathrm{rCBF}$ was almost significantly lower in symptomatic patients $(0.93$ versus 1.03 , $P=.056$ ) (Table 3).

\section{Discussion}

The purpose of this study was to compare how cerebral perfusion is affected by CAS in patients with symptomatic and asymptomatic carotid artery stenosis. First, our results showed a significant improvement after CAS of all 3 perfusion parameters in symptomatic patients, while in asymptomatic patients, only rCBF showed a significant increase. So despite the small number of patients in our study, both symptomatic and asymptomatic patients showed significant improvement of cerebral perfusion parameters after stent placement (Fig 3). When we compared symptomatic and asymptomatic patients before stent placement, rCBF was significantly lower in symptomatic patients. This indicates the presence of more hemodynamic compromise in these patients before treatment of carotid artery stenosis. Finally, rCBV after stent placement was significantly lower in symptomatic patients. In these patients, the compensatory hyperemia on the symptomatic side before treatment $(\mathrm{rCBV}>1)$ turned into hypoxemia after treatment, suggesting impaired autoregulation. These differences in perfusion parameters between both groups of patients before and after stent placement are suggestive of the existence of a different hemodynamic status in symptomatic and asymptomatic patients.

Previous studies have shown differences in hemodynamic status between symptomatic and asymptomatic patients. ${ }^{22,23}$ Silvestrini et $\mathrm{al}^{23}$ described a significant improvement of cerebral hemodynamics in symptomatic patients after CEA by measuring cerebrovascular reactivity. Furthermore, this article reports significant differences in the cerebral hemodynamic perfusion pattern after CEA between symptomatic and asymptomatic patients. ${ }^{23}$ Soinne et $\mathrm{al}^{8}$ investigated patients with asymptomatic and symptomatic stenosis by means of dynamic susceptibility contrast MR imaging and transcranial Doppler sonography. They found a significant difference between symptomatic and asymptomatic patients both before as well in response to CEA. However, it is not yet clear if cerebral perfusion re-establishes equally in surgical patients and those with CAS.

To our knowledge, our study is the first comparing symptomatic and asymptomatic patients with a carotid artery stenosis by using CTP. Our results are in agreement with these above-mentioned previous studies, but we think our study provides additional evidence for the benefit of stent placement in patients with an asymptomatic carotid artery stenosis by showing improvement in both rCBF and dMTT.

In the first stage of hemodynamic compromise, collaterals of the brain are not able to maintain normal cerebral perfusion, which leads to reflex vasodilation and subsequent elevation of intravascular CBV. CBF is still preserved at this stage of hemodynamic compromise. When further reductions in perfusion pressure take place with concurrent increasing MTT, CBV reaches its maximum and cerebrovascular autoregulation is not sufficient to maintain normal perfusion. Stage II hemodynamic failure occurs when CBF declines and oxygen extraction increases. Several studies have tried to determine the association between stage I or II hemodynamic compromise and the risk of stroke. Contradicting results concerning the association between stage I hemodynamic compromise and cerebrovascular events have been reported. ${ }^{3}$ However, stage II hemodynamic compromise has been shown to be an independent predictor of stroke in patients with symptomatic carotid occlusion. ${ }^{24}$ Also patients with symptomatic stenosis and ipsilateral hemodynamic compromise are at higher risk of 
Table 1: Patient characteristics $(n=45)$

\begin{tabular}{|c|c|c|c|}
\hline Patient Demographics & $\begin{array}{c}\text { Symptomatic } \\
\text { Patients }(n=31)\end{array}$ & $\begin{array}{c}\text { Asymptomatic } \\
\text { Patients }(n=14)\end{array}$ & $P$ Value $^{\mathrm{a}}$ \\
\hline Age (yr) (mean) (range) & $67.0 \pm 10.4(43-82)$ & $69.6 \pm 9.0(56-82)$ & $.43^{\mathrm{b}}$ \\
\hline Male (No.) (\%) & $21(67.7)$ & $13(92.9)$ & $.13^{\mathrm{c}}$ \\
\hline \multicolumn{4}{|l|}{ Carotid arteries } \\
\hline Left side treated (No.) (\%) & $15(48.4)$ & $9(64.3)$ & .32 \\
\hline Stenosis grade $(\%)$, mean \pm SD (range) & $89.5 \pm 9.4(65-99)$ & $88.9 \pm 5.8(75-99)$ & $.32^{\mathrm{b}}$ \\
\hline \multicolumn{4}{|l|}{ Symptoms } \\
\hline Stroke (No.) (\%) & $11(35.5)$ & None & N.A. \\
\hline TIA (No.) (\%) & $13(41.9)$ & None & N.A. \\
\hline AF (No.) (\%) & $7(22.6)$ & None & N.A. \\
\hline \multicolumn{4}{|l|}{ Interval in days } \\
\hline Pretreatment CTP to treatment (mean) (range) & $3.2 \pm 6.4(0-35)$ & $3.5 \pm 1.9(3-10)$ & $.03^{\mathrm{b}}$ \\
\hline Treatment to posttreatment CTP (mean) (range) & $33.9 \pm 4.8(22-44)$ & $71.9 \pm 82.6(8-263)$ & $.84^{\mathrm{b}}$ \\
\hline \multicolumn{4}{|l|}{ Medical history } \\
\hline Hypertension (No.) (\%) & $25(80.6)$ & $13(92.9)$ & $.41^{\mathrm{c}}$ \\
\hline Diabetes mellitus (№.) (\%) & $3(9.7)$ & $3(21.4)$ & $.36^{\mathrm{c}}$ \\
\hline Hypercholesterolemia (№.) (\%) & $12(38.7)$ & $12(85.7)$ & .008 \\
\hline Smoking (No.) (\%) & $10(32.3)$ & $6(42.9)$ & $.52^{\mathrm{C}}$ \\
\hline Previous myocardial infarction (№.) (\%) & $4(12.9)$ & $3(21.4)$ & $.66^{\mathrm{c}}$ \\
\hline Previous CABG (№.) (\%) & $2(6.5)$ & $1(7.1)$ & $1.00^{\mathrm{c}}$ \\
\hline
\end{tabular}

Note:-N.A. indicates not applicable; $A E$, atrial fibrillation.

a $P$ values analyzed with the Pearson $\chi^{2}$ test.

b $P$ values analyzed with Mann-Whitney $U$ test.

c $P$ values analyzed with Fisher exact test.

\begin{tabular}{|c|c|c|c|c|c|c|}
\hline Pre- and Posttreatment & $\begin{array}{l}\mathrm{rCBV}(\mathrm{mL} / 100 \mathrm{~g}) \\
\text { (mean) }\end{array}$ & $P$ Value & $\begin{array}{l}\mathrm{dMTT}(\mathrm{s}) \\
\text { (mean) }\end{array}$ & $P$ Value & $\begin{array}{l}\mathrm{rCBF}(\mathrm{mL} / 100 \mathrm{~g} / \mathrm{min}) \\
\text { (mean) }\end{array}$ & $P$ Value \\
\hline Symptomatic patients before treatment $(n=31)$ & $1.02 \pm 0.13$ & & $1.29 \pm 1.21$ & & $0.81 \pm 0.14$ & \\
\hline Symptomatic patients after treatment $(n=31)$ & $0.95 \pm 0.14$ & $.036^{\mathrm{b}}$ & $0.14 \pm 1.08$ & $<.001^{\mathrm{b}}$ & $0.93 \pm 0.17$ & $<.001^{\mathrm{b}}$ \\
\hline Asymptomatic patients before treatment $(n=14)$ & $1.03 \pm 0.08$ & & $0.56 \pm 0.66$ & & $0.92 \pm 0.12$ & \\
\hline Asymptomatic patients, after treatment $(n=14)$ & $1.05 \pm 0.13$ & .221 & $0.06 \pm 0.55$ & 0.081 & $1.03 \pm 0.14$ & $.026^{\mathrm{b}}$ \\
\hline
\end{tabular}

a The significance of the difference between relative perfusion parameters was tested using the paired-samples $t$ test in symptomatic patients and the Wilcoxon signed rank test in asymptomatic patients.

${ }^{\mathrm{b}}$ Significant difference with a $P$ value $<.05$

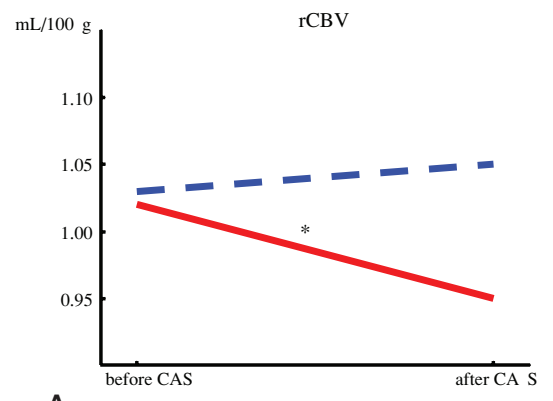

A

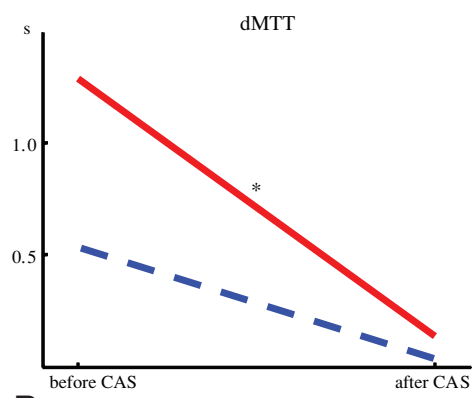

B

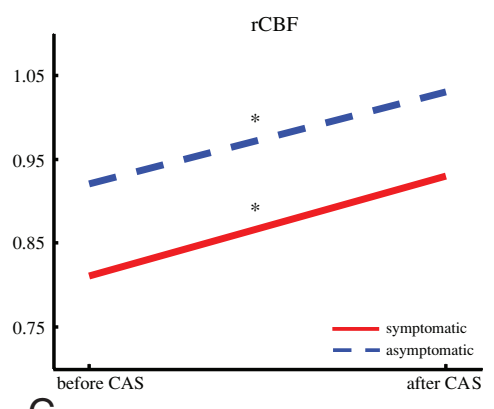

C

Fig 2. Relative CTP values measured before and after treatment in symptomatic and asymptomatic patients: $r C B V(A)$, $d M T T(B)$, and rCBF $(C)$.

Table 3: Comparison between symptomatic and asymptomatic patients in pre- and posttreatment values

\begin{tabular}{|c|c|c|c|c|c|c|}
\hline Pre- and Posttreatment & $\begin{array}{c}\mathrm{rCBV}(\mathrm{mL} / 100 \mathrm{~g}) \\
(\text { mean) }\end{array}$ & $P$ Value & $\begin{array}{l}\mathrm{dMTT}(\mathrm{s}) \\
\text { (mean) }\end{array}$ & $P$ Value & $\begin{array}{c}\mathrm{rCBF}(\mathrm{mL} / 100 \mathrm{~g} / \mathrm{min}) \\
(\text { mean })\end{array}$ & $P$ Value \\
\hline Symptomatic patients before treatment $(n=31)$ & $1.02 \pm 0.13$ & & $1.29 \pm 1.21$ & & $0.81 \pm 0.14$ & \\
\hline Asymptomatic patients before treatment $(n=14)$ & $1.03 \pm 0.08$ & .902 & $0.56 \pm 0.66$ & .061 & $0.92 \pm 0.12$ & $.009^{b}$ \\
\hline Symptomatic patients after treatment $(n=31)$ & $0.95 \pm 0.14$ & & $0.14 \pm 1.08$ & & $0.93 \pm 0.17$ & \\
\hline Asymptomatic patients after treatment $(n=14)$ & $1.05 \pm 0.13$ & $.009^{b}$ & $0.06 \pm 0.55$ & .573 & $1.03 \pm 0.14$ & .056 \\
\hline
\end{tabular}

a The significance of the difference between relative perfusion parameters was tested using the Mann-Whitney $U$ test.

b Significant difference with a $P$ value $<.05$.

disabling stroke than patients with normal cerebral perfusion. ${ }^{4}$

It is important to evaluate individual perfusion parameters and relate these to the different stages of hemodynamic compromise. MTT is considered the most sensitive perfusion parameter because it directly relates to cerebral perfusion pres- 

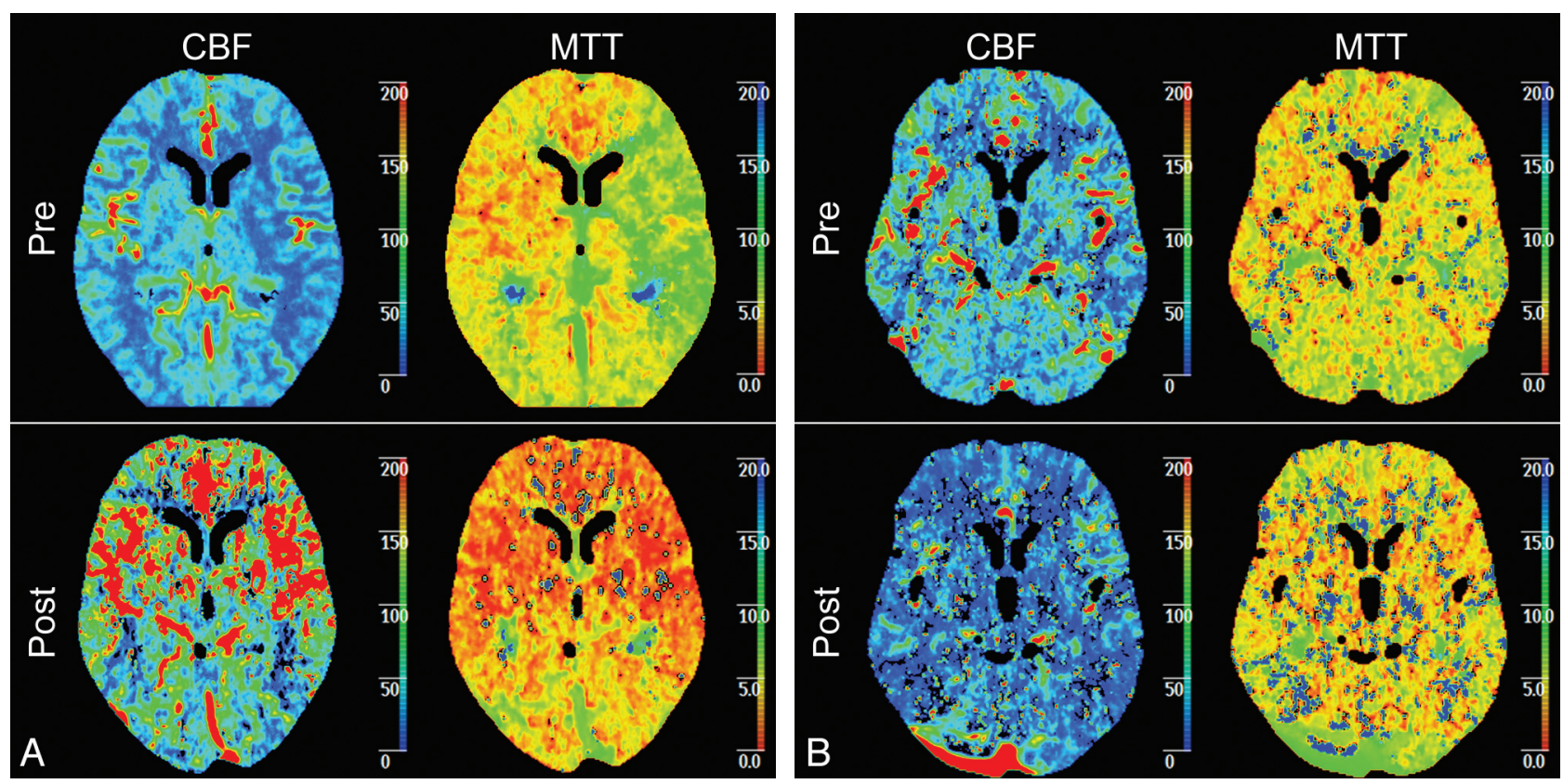

Fig 3. $A$, Example of symptomatic patient with a left-sided carotid artery stenosis of $99 \%$. Before treatment, CBF and MTT show explicit differences between the right and left hemispheres with a higher CBF and a higher MTT in the right hemisphere in comparison with the left hemisphere. After CAS, both CBF and MTT show a symmetric pattern of perfusion. $B$, An example of a asymptomatic patient with a left-sided carotid artery stenosis of $95 \%$. Before treatment, CBF and MTT show differences between the right and left hemisphere with a higher CBF and a higher MTT in the right hemisphere in comparison with the left hemisphere. However, this difference is not as clear as in the symptomatic patient. After CAS, both CBF and MTT show a symmetric pattern of perfusion.

sure. $^{21}$ Before treatment, dMTT was higher in symptomatic patients, though this difference was not significant. dMTT changed significantly after treatment in symptomatic patients, while it did not change significantly in asymptomatic patients. Both findings confirm the presence of differences in cerebral hemodynamics between symptomatic and asymptomatic patients. Also, these results imply that symptomatic patients are more hemodynamically compromised.

CBV remains a parameter that is difficult to interpret, despite extensive studies analyzing it. ${ }^{25}$ It might reflect autoregulatory capacity, because it represents the vasodilatory and vasoconstrictive capacity of the brain. One could expect to observe normalization and symmetry of CBV after treatment, though it is not clear when vasodilation turns into vasoconstriction. Also, it is possible that autoregulation takes more time to recover. The significantly lower rCBV in symptomatic patients might suggest irreversible impaired autoregulation in this group of patients, though a CTP scan should be repeated after 1 year to provide definite conclusions. Thus, we can explain the higher dMTT and lower rCBF in symptomatic patients before treatment by the known hemodynamic responses in the presence of carotid artery stenosis. The increase in perfusion can be a result of loss of normal vasoconstriction secondary to chronic dilation of resistant vessels and impaired cerebral vasoreactivity. The reduced $\mathrm{rCBV}$ in symptomatic patients after treatment is a new finding, which might represent disturbed autoregulatory capacity.

The presence of a hemodynamically significant carotid artery lesion is regarded as one of the possible causes of an impaired cerebral circulation. A stenosis itself, however, is a poor indicator of the hemodynamic status of the cerebral circulation in the ipsilateral hemisphere. ${ }^{26}$ Several important mechanisms are known to maintain normal cerebral perfusion pressure via collaterals of the circle of Willis, ophthalmic collaterals, and leptomeningeal arteries. This study, showing differences of cerebral perfusion between asymptomatic and symptomatic patients, supports the relation between symptoms and impaired cerebral hemodynamics.

Van der Heyden et $\mathrm{al}^{27}$ recently showed that cerebral perfusion parameters improve after carotid stent placement in $50 \%$ of asymptomatic patients. This indicates the presence of a compromised cerebral circulation due to a carotid artery stenosis. CTP analysis can contribute to the selection of patients with a compromised cerebral circulation who would benefit from CAS.

One of the limitations of our study is that we did not compare CTP values to a reference standard, though previous studies have shown that CBF measured with CTP correlates well to PET or xenon-enhanced CT. ${ }^{28}$ Second, we did not evaluate the effects of the configuration of the circle of Willis on perfusion, because for such a multifactorial analysis, more patients are needed. Previous studies have reported conflicting results with regard to the relation between the presence of collaterals and cerebral hemodynamics. Jongen et $\mathrm{al}^{29} \mathrm{re}-$ ported that the presence or absence of collateral pathways in the circle of Willis did not affect perfusion in the ipsilateral MCA territory. Third, symptomatic and asymptomatic patients underwent CTP in different hospitals, though always in multisection CT scanners. To eliminate physiologic variation and interpatient differences, we compared relative values, relating absolute perfusion data in the treated hemisphere to the contralateral and untreated side.

A fourth drawback of this study is a methodologic problem with the deconvolution algorithm. We used a delay-sensitive algorithm, which may underestimate blood flow and overestimate MTT. ${ }^{30}$ However, at this time, clinical experience by 
using these algorithms is not yet available. Another limitation of this study is that arterial input function can have a significant impact on the perfusion parameters. Finally, we could not include as many patients with asymptomatic stenosis as with symptomatic carotid stenosis. However, because treatment of asymptomatic stenosis is still controversial, inclusion of asymptomatic patients for CTP analysis is limited, and these data provide a representative sample of patients with asymptomatic carotid artery stenosis.

\section{Conclusions}

CTP analysis can be used to identify differences between patients with symptomatic and asymptomatic carotid stenosis before revascularization, as well as differences in their response to treatment. Before treatment, symptomatic patients had significantly lower rCBF. After treatment, rCBV was significantly lower in symptomatic patients.

Carotid artery stent placement improves cerebral perfusion in symptomatic and asymptomatic patients. However, cerebral blood flow before treatment is more strongly impaired in patients with symptomatic carotid stenosis. In these patients, the compensatory hyperemia on the symptomatic side before treatment $(\mathrm{rCBV}>1$ ) turns into hypoxemia after treatment, suggesting severely impaired autoregulation. CTP analysis can contribute to the selection of asymptomatic patients with a compromised cerebral circulation who would benefit from CAS.

\section{References}

1. Rothwell PM. Carotid artery disease and the risk of ischaemic stroke and coronary vascular events. Cerebrovasc Dis 2000;10(suppl 5):21-33

2. Golledge J, Greenhalgh RM, Davies AH. The symptomatic carotid plaque. Stroke 2000;31:774-81

3. Derdeyn CP, Grubb RL Jr, Powers WJ. Cerebral hemodynamic impairment: methods of measurement and association with stroke risk. Neurology 1999: 53:251-59

4. Blaser T, Hofmann K, Buerger T, et al. Risk of stroke, transient ischemic attack, and vessel occlusion before endarterectomy in patients with symptomatic severe carotid stenosis. Stroke 2002;33:1057-62

5. Klijn CJ, Kappelle LJ, Tulleken CA, et al. Symptomatic carotid artery occlusion: a reappraisal of hemodynamic factors. Stroke 1997;28:2084-93

6. Grubb RL Jr, Derdeyn CP, Fritsch SM, et al. Importance of hemodynamic factors in the prognosis of symptomatic carotid occlusion. JAMA 1998;280:1055-60

7. Silvestrini M, Vernieri F, Pasqualetti P, et al. Impaired cerebral vasoreactivity and risk of stroke in patients with asymptomatic carotid artery stenosis. JAMA 2000;283:2122-27

8. Soinne L, Helenius J, Tatlisumak T, et al. Cerebral hemodynamics in asymptomatic and symptomatic patients with high-grade carotid stenosis undergoing carotid endarterectomy. Stroke 2003;34:1655-61

9. Rothwell PM, Eliasziw M, Gutnikov SA, et al. Analysis of pooled data from the randomised controlled trials of endarterectomy for symptomatic carotid stenosis. Lancet 2003;361:107-16

10. Inzitari D, Eliasziw $\mathrm{M}$, Gates $\mathrm{P}$, et al. The causes and risk of stroke in patients with asymptomatic internal-carotid-artery stenosis: North American Symptomatic Carotid Endarterectomy Trial Collaborators. N Engl J Med 2000;342:1693-700

11. Endarterectomy for asymptomatic carotid artery stenosis: Executive Committee for the Asymptomatic Carotid Atherosclerosis Study. JAMA 1995;273:1421-28

12. Halliday A, Mansfield A, Marro J, et al. Prevention of disabling and fatal strokes by successful carotid endarterectomy in patients without recent neurological symptoms: randomised controlled trial. Lancet 2004;363:1491-502

13. Hobson RW, Weiss DG, Fields WS, et al. Efficacy of carotid endarterectomy for asymptomatic carotid stenosis: the Veterans Affairs Cooperative Study Group. N Engl J Med 1993;328:221-27

14. Trojanowska A, Drop A, Jargiello T, et al. Changes in cerebral hemodynamics after carotid stenting: evaluation with CT perfusion studies. J Neuroradiol 2006;33:169-74

15. Waaijer A, van Leeuwen MS, van Osch MJ, et al. Changes in cerebral perfusion after revascularization of symptomatic carotid artery stenosis: CT measurement. Radiology 2007;245:541-48

16. Ederle J, Dobson J, Featherstone RL, et al. Carotid artery stenting compared with endarterectomy in patients with symptomatic carotid stenosis (International Carotid Stenting Study): an interim analysis of a randomised controlled trial. Lancet 2010;375:985-97

17. Wintermark M, Smith WS, Ko NU, et al. Dynamic perfusion CT: optimizing the temporal resolution and contrast volume for calculation of perfusion CT parameters in stroke patients. AJNR Am J Neuroradiol 2004;25:720-29

18. Wintermark $M$, Maeder $P$, Verdun FR, et al. Using $80 \mathrm{kVp}$ versus $120 \mathrm{kVp}$ in perfusion CT measurement of regional cerebral blood flow. AJNR Am J Neuroradiol 2000;21:1881-84

19. Cianfoni A, Colosimo C, Basile M, et al. Brain perfusion CT: principles, technique and clinical applications. Radiol Med 2007;112:1225-43

20. Damasio H. A computed tomographic guide to the identification of cerebral vascular territories. Arch Neurol 1983;40:138-42

21. Schumann P, Touzani O, Young AR, et al. Evaluation of the ratio of cerebral blood flow to cerebral blood volume as an index of local cerebral perfusion pressure. Brain 1998;121(pt 7):1369-79

22. Rutgers DR, Klijn CJ, Kappelle LJ, et al. Sustained bilateral hemodynamic benefit of contralateral carotid endarterectomy in patients with symptomatic internal carotid artery occlusion. Stroke 2001;32:728-34

23. Silvestrini M, Troisi E, Matteis M, et al. Transcranial Doppler assessment of cerebrovascular reactivity in symptomatic and asymptomatic severe carotid stenosis. Stroke 1996;27:1970-73

24. Webster MW, Makaroun MS, Steed DL, et al. Compromised cerebral blood flow reactivity is a predictor of stroke in patients with symptomatic carotid artery occlusive disease. J Vasc Surg 1995;21:338-44

25. Derdeyn CP, Videen TO, Yundt KD, et al. Variability of cerebral blood volume and oxygen extraction: stages of cerebral haemodynamic impairment revisited. Brain 2002;125(pt 3):595-607

26. Powers WJ, Press GA, Grubb RL, et al. The effect of hemodynamically significant carotid artery disease on the hemodynamic status of the cerebral circulation. Ann Intern Med 1987;106:27-34

27. Van der Heyden J, Waaijer A, Van EW, et al. CT measurement of changes in cerebral perfusion in patients with asymptomatic carotid artery stenosis undergoing carotid stenting prior to cardiac surgery: “proof of principle.” Eurointervention 2011;6:1091-97

28. Wintermark M, Thiran JP, Maeder P, et al. Simultaneous measurement of regional cerebral blood flow by perfusion CT and stable xenon CT: a validation study. AJNR Am J Neuroradiol 2001;22:905-14

29. Jongen LM, van der Worp HB, et al. Interrelation between the degree of carotid stenosis, collateral circulation and cerebral perfusion. Cerebrovasc Dis 2010;30:277-84

30. Kudo K, Sasaki M, Yamada K, et al. Differences in CT perfusion maps generated by different commercial software: quantitative analysis by using identical source data of acute stroke patients. Radiology 2010;254:200-09 